\title{
Intravitreal Ranibizumab for Diabetic Macular Edema with Prompt versus Deferred Laser Treatment
}

\section{Citation}

Elman, Michael J., Haijing Qin, Lloyd Paul Aiello, Roy W. Beck, Neil M. Bressler, Frederick L. Ferris, Adam R. Glassman, Raj K. Maturi, and Michele Melia. 2012. Intravitreal Ranibizumab for Diabetic Macular Edema with Prompt Versus Deferred Laser Treatment. Ophthalmology 119, no. 11: 2312-2318. doi:10.1016/j.ophtha.2012.08.022.

\section{Published Version}

doi:10.1016/j.ophtha.2012.08.022

\section{Permanent link}

http://nrs.harvard.edu/urn-3:HUL.InstRepos:35772045

\section{Terms of Use}

This article was downloaded from Harvard University's DASH repository, and is made available under the terms and conditions applicable to Other Posted Material, as set forth at http:// nrs.harvard.edu/urn-3:HUL.InstRepos:dash.current.terms-of-use\#LAA

\section{Share Your Story}

The Harvard community has made this article openly available.

Please share how this access benefits you. Submit a story.

Accessibility 


\title{
Intravitreal Ranibizumab for Diabetic Macular Edema with Prompt vs Deferred Laser Treatment: 3-year Randomized Trial Results
}

\author{
Diabetic Retinopathy Clinical Research Network ${ }^{*}$
}

\section{Abstract}

Objective-To report 3-year follow-up within a previously reported randomized trial evaluating prompt versus deferred (for $\geq 24$ weeks) focal/grid laser treatment in eyes treated with intravitreal 0.5 -mg ranibizumab for diabetic macular edema (DME).

Design-Multicenter randomized clinical trial.

Participants-Three hundred and sixty one participants with visual acuity of 20/32 to 20/320 (approximate Snellen equivalent) and DME involving the fovea.

Methods-Ranibizumab every four weeks until no longer improving (with resumption if worsening) and random assignment to focal/grid laser treatment promptly or deferred ( $\geq 24$ weeks).

Main Outcome Measures-Best-corrected visual acuity and safety at the 156-week ("3-year") visit.

Results-The estimated mean change in visual acuity letter score from baseline through the 3year visit was 2.9 letters greater $(9.7$ versus 6.8 , mean difference $=2.9,95 \%$ confidence interval 0.4 to $5.4, P=0.02$ ) in the deferral group compared with the prompt laser treatment group. In the prompt laser treatment group and deferral group, respectively, the percentage of eyes with a $\geq 10$

(C) 2012 American Academy of Ophthalmology, Inc. Published by Elsevier Inc. All rights reserved.

Corresponding author: Haijing Qin, Jaeb Center for Health Research, 15310 Amberly Drive, Suite 350, Tampa, FL 33647; Phone: (813) 975-8690, Fax: (800) 816-7601, drcrstat3@ jaeb.org.

*A published list of the Diabetic Retinopathy Clinical Research Network investigators and staff participating in this protocol can be found in Ophthalmology 2010;117:1064-1077.e35 with a current list available at www.drcr.net

Financial Disclosures: The funding organization (National Institutes of Health) participated in oversight of the conduct of the study and review of the manuscript but not directly in the design or conduct of the study, nor in the collection, management, analysis, or interpretation of the data, or in the preparation of the manuscript. Genentech provided the ranibizumab for the study. In addition, Genentech provided funds to DRCR.net to defray the study's clinical site costs. As described in the Diabetic Retinopathy Clinical Research Network (DRCR.net) Industry Collaboration Guidelines (available at www.drcr.net), the DRCR.net had complete control over the design of the protocol, ownership of the data, and all editorial content of presentations and publications related to the protocol. A complete list of all DRCR.net investigator financial disclosures can be found at www.drcr.net. Writing Committee financial disclosures: Neil M. Bressler: Grants to investigators at The Johns Hopkins University are negotiated and administered by the institution (such as the School of Medicine) which receives the grants, typically through the Office of Research Administration. Individual investigators who participate in the sponsored project(s) are not directly compensated by the sponsor, but may receive salary or other support from the institution to support their effort on the projects(s). Dr. Neil Bressler is Principal Investigator of grants at The Johns Hopkins University sponsored by the following entities (not including the National Institutes of Health): Abbott Medical Optics, Allergan, Bausch \& Lomb, Bristol-Meyer-Squibb, Carl Zeiss Meditec, EMMES Corporation, ForSight Labs, LLC Genentech, Genzyme Corporation, Lumenis, Notal Vision, Novartis, and Regeneron. A complete list of all DRCR.net investigator financial disclosures can be found at www.drcr.net.

Publisher's Disclaimer: This is a PDF file of an unedited manuscript that has been accepted for publication. As a service to our customers we are providing this early version of the manuscript. The manuscript will undergo copyediting, typesetting, and review of the resulting proof before it is published in its final citable form. Please note that during the production process errors may be discovered which could affect the content, and all legal disclaimers that apply to the journal pertain. 
letter gain was $42 \%$ and $56 \%(P=0.02)$, while the percentage of eyes with a $\geq 10$ letter loss was $10 \%$ and $5 \%(P=0.12)$. Up to the 3 -year visit, the median numbers of injections were 12 and 15 in the prompt and deferral groups, respectively $(P=0.007)$, including 1 and 2 , respectively, from the 2 - up to the 3-year visit. At the 3-year visit, the percentages of eyes with central subfield thickness $\geq 250 \mu \mathrm{m}$ on time domain optical coherence tomography were $36 \%$ in both groups $(P=$ 0.90). In the deferral group, $54 \%$ did not receive laser treatment during the trial. Systemic adverse events appeared similar in the two groups.

Conclusions-These 3-year results suggest that focal/grid laser treatment at the initiation of intravitreal ranibizumab is no better, and possibly worse for vision outcomes, than deferring laser treatment for $\geq 24$ weeks in eyes with DME involving the fovea and with vision impairment.

Some of the observed difference in visual acuity at three years may be related to fewer cumulative ranibizumab injections during follow-up in the prompt laser treatment group. Follow-up through five years continues.

In a comparative effectiveness randomized clinical trial conducted by the Diabetic Retinopathy Clinical Research Network (DRCR.net) study participants with center involved diabetic macular edema (DME), were assigned randomly to intravitreal 0.5-mg ranibizumab combined with prompt or deferred ( $\geq 24$ weeks) focal/grid laser treatment, 4-mg triamcinolone combined with prompt focal/grid laser treatment, or sham injections with prompt focal/grid laser treatment. ${ }^{1}$ The rationale behind the prompt laser treatment strategy was to determine if adding the anti-permeability effects of an anti-vascular endothelial growth factor (anti-VEGF) regimen when applying focal/grid laser treatment provided superior vision outcomes compared with focal/grid laser treatment alone. In addition, it was hypothesized that combining anti-VEGF treatment with prompt laser might have other advantages, such as a prompt reduction of edema from the anti-VEGF agent, which would provide a more immediate improvement in vision and the anatomic and functional benefits of laser treatment that may take several months to be achieved. Furthermore, it was hypothesized that the effects of laser treatment might be longer lasting than those of antiVEGF therapy, thereby reducing the number of injections required to have a sustained effect on resolution of edema. However, if ranibizumab + prompt laser treatment were found to be superior to prompt laser treatment alone, the superior outcomes could be solely due to the anti-VEGF therapy or the combination of the anti-VEGF therapy plus laser treatment. To try to determine if anti-VEGF therapy alone might be superior to laser treatment alone, this protocol included a group of participants randomized to anti-VEGF therapy in which focal/ grid laser treatment was deferred for at least 24 weeks, and only added at the 24-week visit or thereafter if DME persisted and was not improving despite injections of ranibizumab every four weeks. The deferral was a minimum of 24 weeks so that an eye that did not have resolution of DME with anti-VEGF therapy by that time, or some time thereafter, could receive focal/grid laser treatment, which was known to be beneficial, compared with observation for DME. The study found that intravitreal ranibizumab with prompt or deferred laser treatment was more effective through at least two years compared with prompt laser treatment alone for the treatment of DME involving the central macula; ${ }^{2}$ although rarely associated with endophthalmitis. Based on these results, the study was extended for each willing participant from the preplanned three years of follow-up to five years. Only eyes originally assigned to ranibizumab + prompt or deferred laser treatment were continued on their original randomization treatment protocol. Eyes originally assigned to sham plus prompt laser treatment or triamcinolone plus prompt laser treatment were given the opportunity to receive intravitreal ranibizumab. This report compares visual acuity and optical coherence tomography (OCT) outcomes through three years among eyes originally assigned randomly to one of the ranibizumab groups to gain insight into the longer term course of DME treated with ranibizumab in combination with prompt versus deferred laser treatment. 


\section{Methods}

The study procedures and statistical methods have been reported ${ }^{1}$ and are not repeated herein. The study adhered to the tenets of the Declaration of Helsinki. The protocol and Health Insurance Portability and Accountability Act compliant informed consent forms were approved by multiple institutional review boards. The protocol is available on the DRCR.net website (www.drcr.net, date accessed: July 28, 2012). In brief, the trial included participants with visual acuity (approximate Snellen equivalent) of 20/32 to 20/320 (median baseline visual acuity approximately 20/50) and DME involving the fovea (mean OCT central subfield thickness $405 \mu \mathrm{m}$ on time domain OCT). Nearly one-half of study eyes was assigned to ranibizumab + prompt laser treatment or ranibizumab + deferred laser treatment. Ranibizumab was applied every 4 weeks for at least 4 doses; an additional dose was given at weeks 16 and 20 unless success criteria were met (visual acuity Snellen equivalent 20/20 or better, or OCT CSF $<250 \mathrm{um}$ ). Thereafter, ranibizumab generally was given every 4 weeks if the eye improved (OCT CSF decrease by $\geq 10 \%$ or visual acuity increase by $\geqq 5$ letters) compared with the last injection and did not yet meet success criteria; otherwise retreatment was at the discretion of the investigator. Focal/grid laser treatment was applied either promptly ( 7 to 10 days after the initial ranibizumab injection) or deferred for at least 24 weeks. If laser treatment was deferred for at least 24 weeks, it only was given if the eye was not improving from injection alone and there was clinically significant macular edema. Once laser treatment was initiated (either promptly or deferred), it was repeated as often as every 13 weeks if there was clinically significant macular edema and complete laser had not been given. After the 52-week visit, subsequent visit interval could be extended to 8 weeks if injections could be deferred at three consecutive visits; subsequently, if ranibizumab was not resumed after 8 weeks, the follow-up visit interval could be extended to every 16 weeks until ranibizumab was resumed. A longitudinal analysis of visual acuity and OCT central subfield thickness change from baseline for the 16- through the 156-week visits using continuous time models assuming a linear relationship over time, and adjusting for baseline visual acuity or OCT central subfield thickness, was performed on all eyes randomized to the two ranibizumab groups. ${ }^{3}$ Visual acuity changes were truncated to \pm 30 letters to minimize the effects of outliers. All efficacy and safety data through the 3-year visit are reported.

\section{Results}

One-hundred forty-four (80\%) of the ranibizumab + prompt laser treatment and $147(81 \%)$ of the ranibizumab + deferred laser treatment groups completed the 3 -year visit. Without deaths, the 3 -year completion rate was $85 \%$ and $89 \%$, respectively, in the prompt and deferral groups. There was a higher percentage of eyes with prior treatment for diabetic retinopathy, especially prior panretinal photocoagulation, and a higher percentage of eyes with milder levels of diabetic retinopathy in those that completed the 3-year visit compared with those who did not complete that visit (Table 1, available at http://aaojournal.org). Among participants who completed the 3-year visit, no substantial differences were identified when comparing the baseline characteristics between the two ranibizumab groups (Table 2, available at http://aaojournal.org).

Between the 2- and 3-year visits, in the ranibizumab with prompt or deferred laser treatment arms, the median numbers of injections were one and two out of a potential maximum of 13 , respectively. Out of a potential maximum cumulative number of 39 injections during the three years of the study, the median was 12 and 15 in the prompt and deferral groups, respectively. The median number of follow-up visits between years two and three was seven and eight, respectively in each treatment group (Table 3) for a median total of 28 and 30 visits over three years in the prompt and deferral groups. By the 2 -year visit, $43 \%$ of the 
eyes in the ranibizumab + deferred laser treatment group had received at least one session of focal/grid laser treatment; by the 3-year visit, an additional $3 \%$ totaling $46 \%$ had received at least one session of focal/grid laser treatment during the study (Table 3). Between the 2- and 3 -year visits, $25 \%$ in the ranibizumab + prompt laser treatment group and $14 \%$ in the ranibizumab+deferred laser treatment group received at least one session of focal/grid laser treatment. Compliance with the treatment protocol for receipt of ranibizumab injections was excellent with $95 \%$ and $93 \%$ in the ranibizumab + prompt laser treatment and ranibizumab + deferred laser treatment, respectively, receiving an intravitreal ranibizumab injection when required by the protocol between the 2- and 3-year visits.

Figure 1 shows the mean visual acuity change from baseline over time for all randomized study participants in the ranibizumab groups. At the 3-year visit, the estimated mean difference in the visual acuity letter score change from baseline using longitudinal analyses was 2.9 (95\% confidence interval $[\mathrm{CI}] 0.4$ to 5.4$)$ letters less (6.8 versus $9.7, P=0.02)$ in the ranibizumab + prompt laser treatment group compared with the ranibizumab + deferred laser treatment group (Table 4). These mean changes were associated with a greater chance of at least moderate improvement and a smaller chance of substantial loss in visual acuity from baseline in eyes assigned to ranibizumab + deferred laser treatment compared with ranibizumab + prompt laser treatment (Table 5). The percentage of eyes with $\geq 10$ letter gain was $42 \%$ in the ranibizumab + prompt laser treatment compared with $56 \%$ in the ranibizumab+deferred laser treatment group $(P=0.02)$, while the percentage of eyes with $\geq 10$ letter loss was $10 \%$ with ranibizumab + prompt laser treatment compared with $5 \%$ with ranibizumab + deferred laser treatment $(P=0.12)$. Results appeared similar when the analysis was limited to the 291 eyes with 3-year follow-up data (Figure 2, available at http:// aaojournal.org). The treatment effect was consistent with the overall results among various subgroups (Table 6, available at http://aaojournal.org).

OCT data did not appear to mirror the visual acuity differences noted between the ranibizumab groups when looking at Figure 3 and Table 7. For example, at the 3 -year visit, the percentages of eyes with central subfield thickness $2250 \mu \mathrm{m}$ on time domain OCT were $36 \%$ in both groups. Similarly, when a longitudinal analysis of OCT outcomes was performed (Table 8 , available at http://aaojournal.org), little difference at the 3-year visit was apparent when comparing the ranibizumab + prompt laser treatment group to the ranibizumab + deferred laser treatment group.

No differences by treatment group of systemic events attributable to study treatment were apparent (data not shown). One eye (0.5\%) of 187 participants and two eyes (1\%) of 188 participants in the ranibizumab + prompt and ranibizumab + deferred laser treatment groups, respectively, experienced injection-related endophthalmitis.

\section{Discussion}

The 3-year results from this randomized clinical trial comparing two different strategies for treating DME involving the fovea with vision impairment using ranibizumab suggest that adding focal/grid laser treatment at the initiation of intravitreal ranibizumab is no better, and possibly worse for vision outcomes, than deferring addition of laser treatment for at least 24 weeks. Some of the observed difference in visual acuity at three years may be related to the fewer ranibizumab injections during follow-up in the prompt laser treatment group. Investigators were not masked as to whether each study participant was randomly assigned to ranibizumab + prompt laser treatment or ranibizumab + deferred laser treatment. It is possible that the investigator was biased to believe if laser treatment was given then perhaps an injection could be withheld at visits that allowed discretion, leading to fewer injections in the ranibizumab + prompt laser treatment group and worse visual acuity than if more 
injections were given in the prompt laser treatment group. The potentially destructive effects of the laser treatment also may be related to some of the visual acuity differences between groups. Subgroup analyses suggested that outcomes still favored deferred laser of whether the eye received laser treatment for DME prior to study enrollment and initiation of ranibizumab for DME. Regardless of these considerations, the protocol was not designed to permit an evaluation of the relationship between the number of injections or laser treatment sessions or laser treatment spots and visual acuity outcomes.

Another theoretical possibility to explain for the observed differences in visual acuity outcomes could be worsening of retinopathy more frequently in the ranibizumab + prompt laser group compared with the deferred laser group. Worsening of retinopathy through 3 years by treatment arm is the topic of another publication in preparation by the DRCR.net at this time. Nevertheless, data from analyses for that publication suggest that the number of vitreous hemorrhages in each ranibizumab arm are too few, and the differences between the treatment arms for this event are too small to account for the visual acuity differences reported in this manuscript.

The differences noted between the prompt and deferral groups were consistent over time from week 52 to week 156, except at the 120-week follow-up visit when the differences between the groups appeared minimal. It is unknown why this might have occurred only at this one time point. Post-hoc analyses performed to try to identify reasons for this finding other than chance included an analysis of: (1) whether there were differences at that visit due to deaths or other sources of missing data; (2) patient characteristics for those seen at the week-120 visit compared to those seen at the 104-week visit or 136-week visit, and (3) distribution of time since last injection prior to the 120 -week visit. None of these evaluations appeared to explain the inconsistent finding between groups at the 120-week follow-up visit.

No differences by treatment group of systemic events attributable to study treatment were apparent. Differences seem unlikely given that both groups were receiving ranibizumab.

These DRCR.net results extend the results from two years regarding the continued decreased frequency of intravitreal injections applied while maintaining the visual acuity outcomes noted at one and two years following initiation of ranibizumab treatment. A median of six injections for the first six months, three injections in the second six months, two to three injections in the second year, and one to two injections in the third year were observed. Despite the decreasing number of injections given in the second and third year of management, the ranibizumab + deferred laser treatment group showed no decline in visual acuity when every 4 week ranibizumab no longer was required starting at the week 16 to 24 visit, and the ranibizumab + prompt laser treatment group showed only a slight decline from the 1-year to 3-year visit.

The finding that the beneficial effect on visual acuity apparent within the first six months of treatment continued over at least three years of follow-up despite a steadily decreasing number of injections of ranibizumab suggests that this treatment protocol is not just transiently blocking the effects of VEGF on macular edema. Rather, the treatment might be having a more fundamental effect on the basic mechanism(s) of the disease, i.e., the mechanism(s) by which VEGF is formed, secreted, degraded or its efficiency in stimulating a response. Another possibility is that DME due to VEGF has a limited life span of activity and can resolve after one to two years in many cases. The anti-VEGF therapy theoretically can prevent damage during this period of time and then no longer is required as frequently as the production of VEGF diminishes. The decreasing need for treatment while visual acuity benefit is maintained leads to several questions for future investigations evaluation. Long term clinical trial results indicate that the required frequency of intravitreal anti-VEGF 
injections is higher, on average, for choroidal neovascularization in AMD than for DME. ${ }^{4}$ (Heier, J.S. 96 weeks results from the VIEW 1 and VIEW 2 studies: Intravitreal Aflibercept injection versus Ranibizumab for neovascular AMD shows sustained improvements in visual acuity. Paper presented at ARVO Annual Meeting, May 10, 2012; Ft. Lauderdale) Further studies are necessary to evaluate whether this is a specific drug effect on the disease or a difference in the natural history of retinal vascular diseases.

In summary, although fewer injections are likely to be given when prompt laser treatment is included with initiation of ranibizumab, these results suggest that such a strategy is no better, and possibly worse at three years, than deferring addition of laser treatment for at least 24 weeks in eyes with diabetic macular edema involving the fovea with vision impairment. Of note, there was no group receiving intravitreal ranibizumab without the option of receiving prompt or deferred focal/grid laser treatment to determine if a strategy of never allowing laser treatment would have been equally effective. Follow-up through five years continues for this protocol. While these results are based on outcomes of a group, individual patients may benefit from other strategies based on individual decisions by the patient and physician.

\section{Supplementary Material}

Refer to Web version on PubMed Central for supplementary material.

\section{Acknowledgments}

Financial Support: Supported through cooperative agreements from the National Eye Institute and the National Institute of Diabetes and Digestive and Kidney Diseases, National Institutes of Health, Department of Health and Human Services EY14231, EY14229, EY18817

\section{References}

1. Elman MJ, Aiello P, Beck RW, et al. Diabetic Retinopathy Clinical Research Network. Randomized trial evaluating ranibizumab plus prompt or deferred laser or triamcinolone plus prompt laser for diabetic macular edema. Ophthalmology. 2010; 117:1064-77. [PubMed: 20427088]

2. Elman MJ, Bressler NM, Qin H, et al. Diabetic Retinopathy Clinical Research Network. Expanded 2-year follow-up of ranibizumab plus prompt or deferred laser or triamcinolone plus prompt laser for diabetic macular edema. Ophthalmology. 2011; 118:609-14. [PubMed: 21459214]

3. Verbeke, G.; Molenberghs, G. Linear Mixed Models for Longitudinal Data. New York: Springer; 2000. p. 55-76.Springer Series in Statistics

4. Martin DF, Maguire MG, Fine SL, et al. Comparison of Age-related Macular Degeneration Trials (CATT) Research Group. Ranibizumab and bevacizumab for treatment of neovascular age-related macular degeneration: two-year results. Ophthalmology. 2012; 119:1388-98. [PubMed: 22555112]

\section{Writing Committee}

${ }^{1}$ Michael J. Elman, MD; ${ }^{2}$ Haijing Qin, MS; ${ }^{3}$ Lloyd Paul Aiello; ${ }^{2}$ Roy W. Beck, MD; ${ }^{4}$ Neil M. Bressler, MD; ${ }^{5}$ Frederick L. Ferris III, MD; ${ }^{2}$ Adam R. Glassman, MS; ${ }^{6}$ Raj K. Maturi, MD; ${ }^{2}$ Michele Melia, ScM.

\footnotetext{
${ }^{1}$ Elman Retina Group, PA;

2 Jaeb Center for Health Research;

3 Beetham Eye Institute, Joslin Diabetes Center, Department of Ophthalmology, Harvard Medical School;

${ }^{4}$ Wilmer Eye Institute, Johns Hopkins University School of Medicine;

${ }^{5}$ National Eye Institute and the National Institute of Health;

$6_{\text {Raj K. Maturi, M.D., P.C. }}$
} 


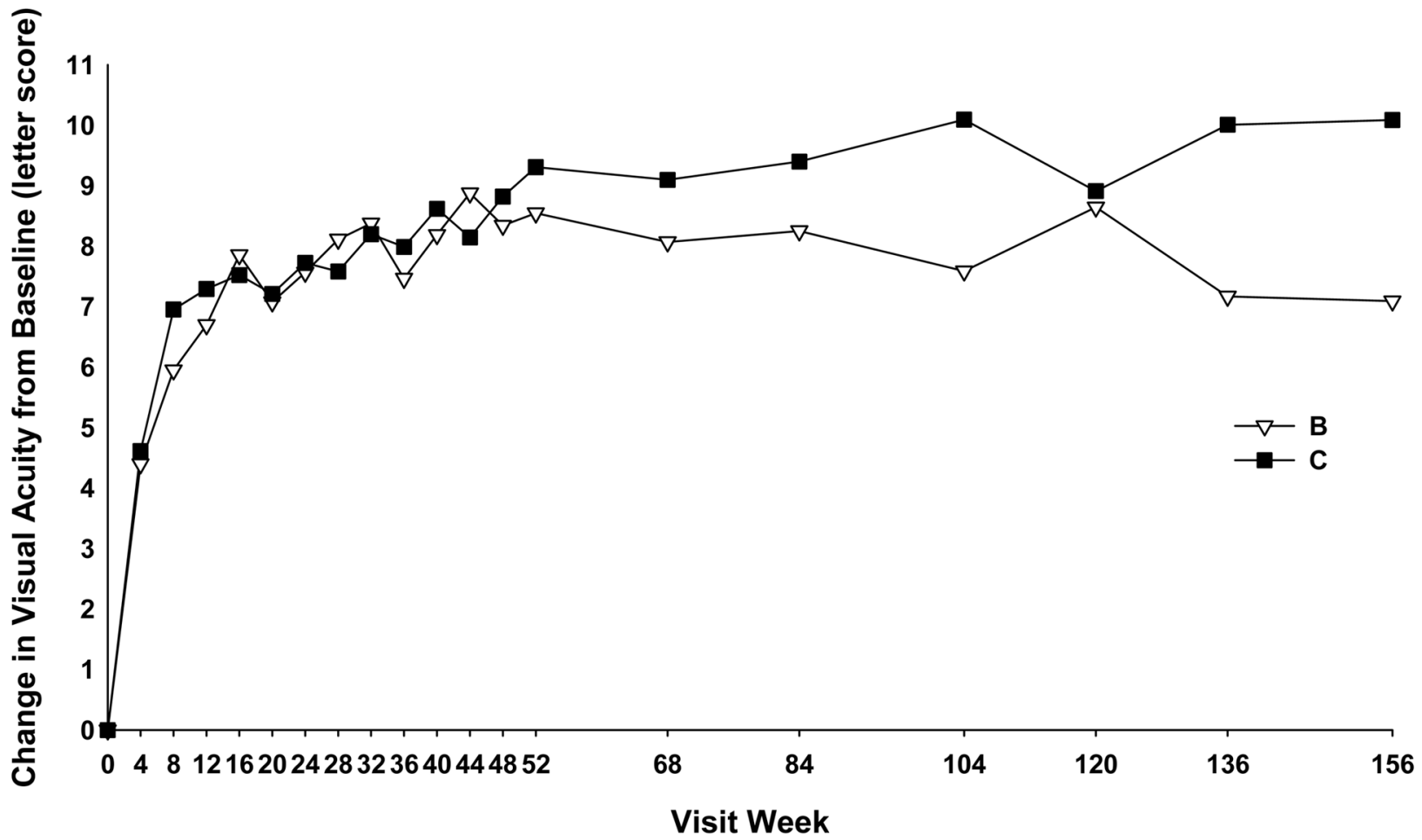

Open triangle $(B)=$ Ranibizumab + prompt laser treatment; closed square $(C)=$ Ranibizumab + deferred laser treatment

$\begin{array}{lccccccc} & \mathbf{5 2} & \mathbf{6 8} & \mathbf{8 4} & \mathbf{1 0 4} & \mathbf{1 2 0} & \mathbf{1 3 6} & \begin{array}{c}156 \\ \text { weeks } \\ \text { weeks }\end{array} \\ \begin{array}{l}\text { weeks } \\ \text { treatment, N }\end{array} & 165 & 157 & 155 & 156 & 143 & 140 & 144 \\ \begin{array}{l}\text { Ranibizumab + deferred Laser } \\ \text { treatment, N }\end{array} & 173 & 167 & 160 & 161 & 149 & 143 & 147\end{array}$

Figure 1. Mean Change in Visual Acuity at Follow-up Visits

Values that were \pm 30 letters were assigned a value of 30 . Results were similar without truncation (data not shown). $P$ value for difference in mean change in visual acuity from ranibizumab + prompt laser treatment (open triangle) at the 156-week study visit for ranibizumab + deferred laser treatment $($ closed square $)=0.02$. Each visit week includes visits that are \pm 14 days, except the $52,68,84,120,136$-week visits that are \pm 8 weeks, and the 104,156 -week visits that are \pm 16 weeks. 


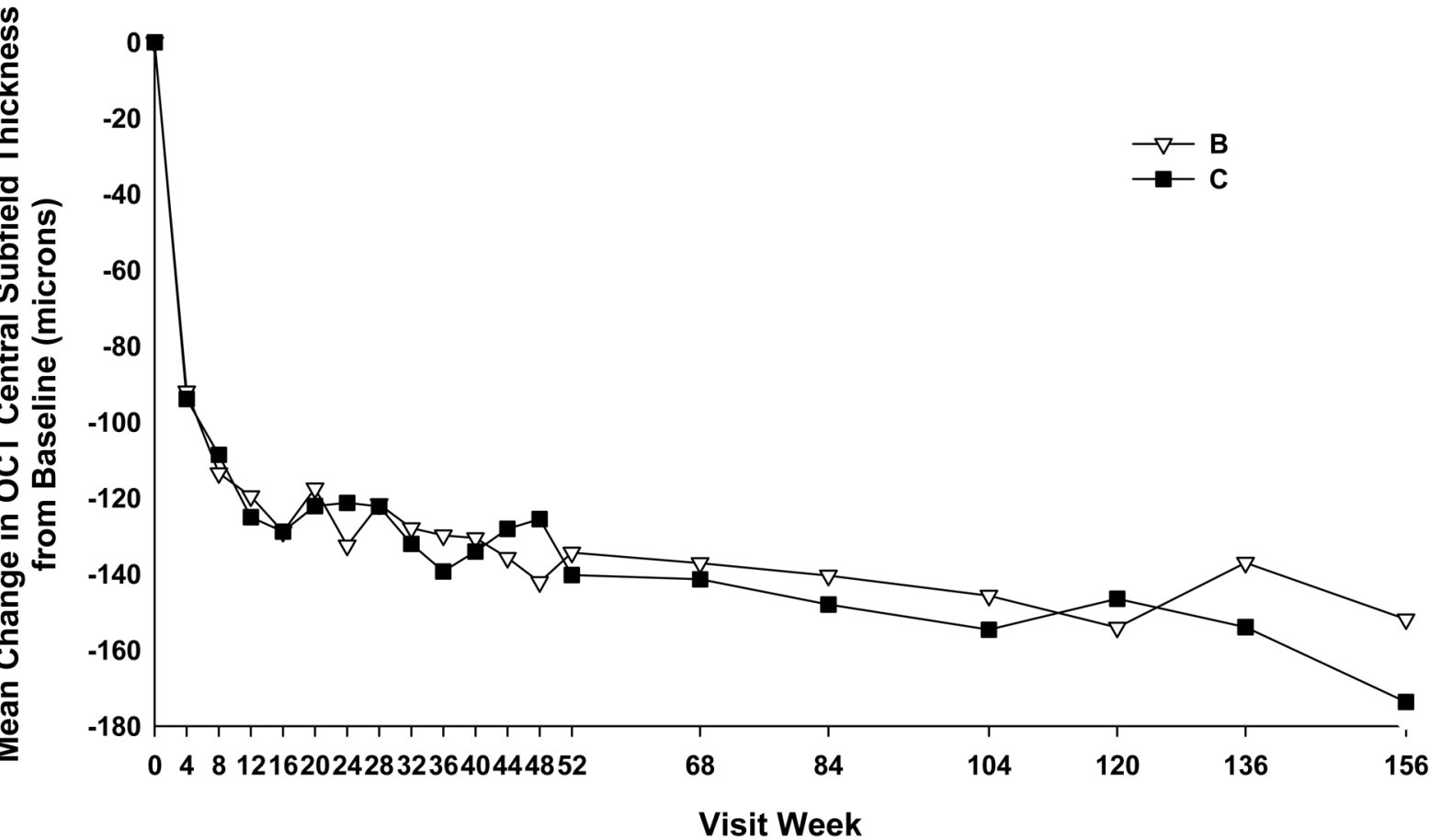

Open triangle $(B)=$ Ranibizumab + prompt laser treatment; closed square $(C)=$ Ranibizumab + deferred laser treatment

Ranibizumab + prompt Laser treatment, $\mathbf{N}$

Ranibizumab + deferred Laser treatment, $\mathbf{N}$

$\begin{array}{ccccccc}\begin{array}{c}\mathbf{5 2} \\ \text { weeks }\end{array} & \begin{array}{c}\mathbf{6 8} \\ \text { weeks }\end{array} & \begin{array}{c}\mathbf{8 4} \\ \text { weeks }\end{array} & \begin{array}{c}\mathbf{1 0 4} \\ \text { weeks }\end{array} & \begin{array}{c}\mathbf{1 2 0} \\ \text { weeks }\end{array} & \begin{array}{c}\mathbf{1 3 6} \\ \text { weeks }\end{array} & \begin{array}{c}\mathbf{1 5 6} \\ \text { weeks }\end{array} \\ 165 & 155 & 154 & 152 & 140 & 131 & 131 \\ 169 & 164 & 156 & 153 & 141 & 133 & 128\end{array}$

Figure 3. Mean Change in Optical Coherence Tomography Central Subfield Retinal Thickening at Follow-up Visits

$P$ value for difference in mean change in optical coherence tomography (OCT) central subfield retinal thickness from ranibizumab + prompt laser treatment (open triangle) at the 156-week study visit for ranibizumab + deferred laser treatment (closed square) $=0.94$.

Each visit week includes visits that are \pm 14 days, except the 52, 68, 84, 120, 136-week visits that are \pm 8 weeks, and the 104,156 -week visits that are \pm 16 weeks. 
Table 3

Visits and Treatments Prior to Three Year Visit ${ }^{*}$ - Median (quartiles) or N (\%)

\begin{tabular}{|c|c|c|}
\hline & $\begin{array}{l}\text { Ranibizumab + Prompt } \\
\text { Laser treatment } N=144\end{array}$ & $\begin{array}{l}\text { Ranibizumab + Deferred } \\
\text { Laser treatment } N=147\end{array}$ \\
\hline Number of visits in year one & $13(12,13)$ & $13(12,13)$ \\
\hline Number of visits in year two & $8(6,11)$ & $10(6,12)$ \\
\hline Number of visits in year three & $7(4,9)$ & $8(4,11)$ \\
\hline Number of visits prior to three year visit & $28(22,32)$ & $30(24,34)$ \\
\hline Number of injections in year one & $8(7,11)$ & $9(7,11)$ \\
\hline Number of injections in year two & $2(0,5)$ & $3(1,7)$ \\
\hline 0 & $45(31 \%)$ & $30(20 \%)$ \\
\hline $1-2$ & $41(28 \%)$ & $33(22 \%)$ \\
\hline $3-5$ & $28(19 \%)$ & $40(27 \%)$ \\
\hline $6-8$ & $17(12 \%)$ & $21(14 \%)$ \\
\hline$\geq 9$ & $13(9 \%)$ & $23(16 \%)$ \\
\hline Number of injections in year three & $1(0,3)$ & $2(0,5)$ \\
\hline 0 & $62(43 \%)$ & $48(33 \%)$ \\
\hline $1-2$ & $39(27 \%)$ & $31(21 \%)$ \\
\hline $3-5$ & $31(22 \%)$ & $37(25 \%)$ \\
\hline $6-8$ & $9(6 \%)$ & $20(14 \%)$ \\
\hline$\otimes 9$ & $3(2 \%)$ & $11(7 \%)$ \\
\hline Number of injections prior to 3-year visit & $12(8,17)$ & $15(9,22)$ \\
\hline $\begin{array}{l}\text { Number }(\%) \text { of eyes that met success }{ }^{\dagger} \text { at } 1 \text {-year visit and then received injection } \\
\text { by } 2 \text {-year visit }\end{array}$ & $52(60 \%)$ of 86 & $46(67 \%)$ of 69 \\
\hline $\begin{array}{l}\text { Number }(\%) \text { of eyes that met success }{ }^{\dagger} \text { at } 2 \text {-year visit and then received injection } \\
\text { by } 3 \text {-year visit }\end{array}$ & $43(47 \%)$ of 92 & $46(53 \%)$ of 87 \\
\hline Number of focal/grid laser treatments from baseline to (prior to) 3-year visit & $3(2,4)$ & $0(0,2)$ \\
\hline $\begin{array}{l}\text { Number (\%) of eyes that received focal/grid laser treatments from baseline to } \\
\text { (prior to) 1-year visit }\end{array}$ & $144(100 \%)$ & $47(32 \%)$ \\
\hline $\begin{array}{l}\text { Number (\%) of eyes that received focal/grid laser treatments from baseline to } \\
\text { (prior to) 2-year visit }\end{array}$ & $144(100 \%)$ & $63(43 \%)$ \\
\hline $\begin{array}{l}\text { Number (\%) of eyes that received focal/grid laser treatments from baseline to } \\
\text { (prior to) 3-year visit }\end{array}$ & $144(100 \%)$ & $68(46 \%)$ \\
\hline $\begin{array}{l}\text { Number (\%) of eyes that did not receive focal/grid laser treatments from 1-year } \\
\text { to (prior to) 2-year visit }\end{array}$ & $83(58 \%)$ & $108(73 \%)$ \\
\hline $\begin{array}{l}\text { Number (\%) of eyes that did not receive focal/grid laser treatments from 2-year } \\
\text { to (prior to) 3-year visit }\end{array}$ & $108(75 \%)$ & $126(86 \%)$ \\
\hline \multicolumn{3}{|c|}{$\begin{array}{l}\text { Includes study participants completing the } 3 \text {-year (156-week) visit; the } 1 \text {-year visit includes visits that occur between } 308 \text { and } 420 \text { days (between } \\
44 \text { and } 60 \text { weeks) from randomization, the 2-year visit includes visits that occur between } 616 \text { and } 840 \text { days (between } 88 \text { and } 120 \text { weeks) from } \\
\text { randomization, and the } 3 \text {-year visit includes visits that occur between } 980 \text { and } 1204 \text { days (between } 140 \text { and } 172 \text { weeks) from randomization. When } \\
\text { more than } 1 \text { visit occurred in the window, data from the visit closest to the target dates were used. }\end{array}$} \\
\hline
\end{tabular}


Page 10
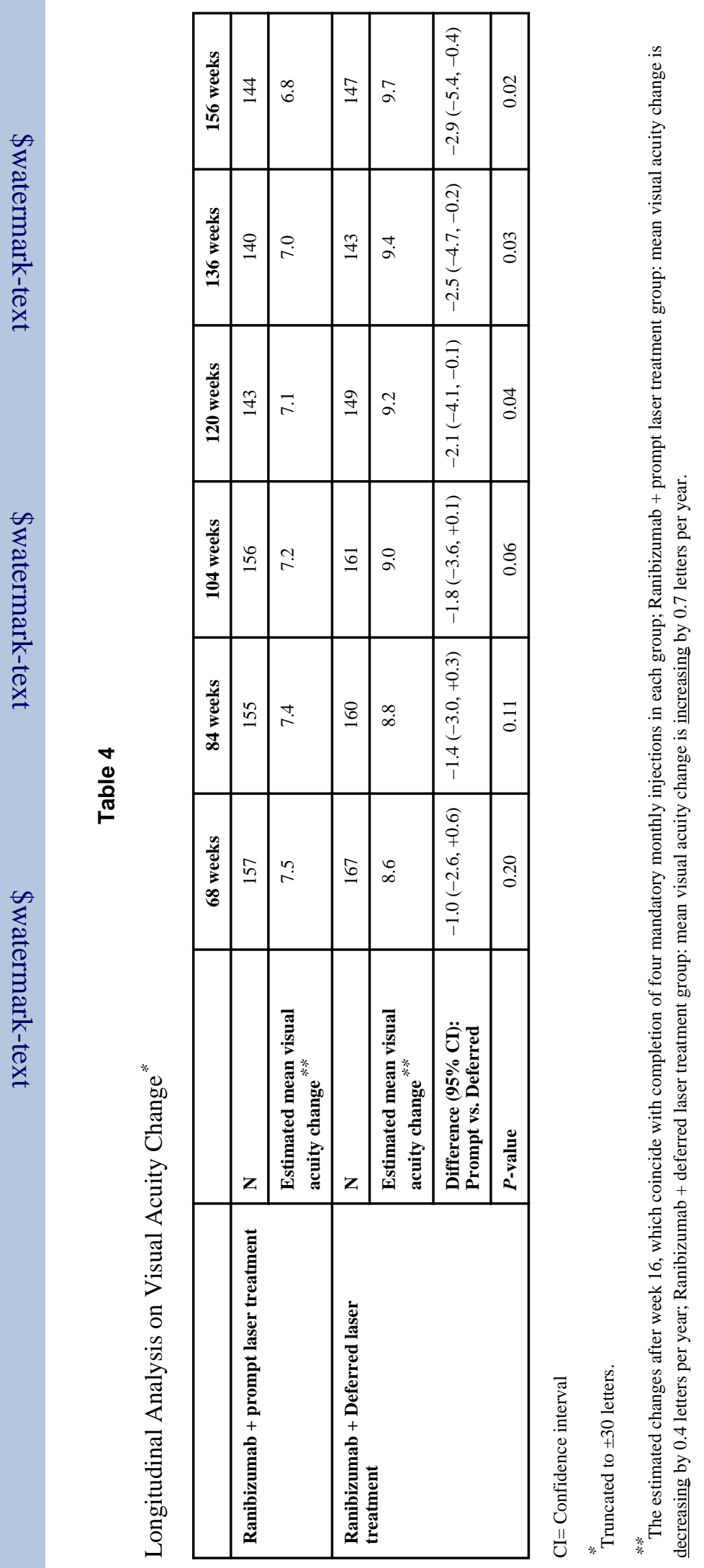

Ophthalmology. Author manuscript; available in PMC 2013 November 01. 
Table 5

Visual Acuity Outcomes from Baseline to the Three Year Visit*

\begin{tabular}{|c|c|c|}
\hline & $\begin{array}{l}\text { Ranibizumab + Prompt } \\
\text { Laser treatment } N=144\end{array}$ & $\begin{array}{l}\text { Ranibizumab + Deferred Laser } \\
\text { treatment } N=147\end{array}$ \\
\hline \multicolumn{3}{|l|}{ Change in visual acuity ${ }^{\dagger}$ (letter score) } \\
\hline Mean \pm SD & $+7 \pm 12$ & $+10 \pm 11$ \\
\hline Median (25th, 75th percentile) & $+8(+2,+16)$ & $+11(+4,+17)$ \\
\hline $\begin{array}{l}\text { Difference in mean change from ranibizumab + prompt laser } \\
\text { treatment }(95 \% \mathrm{CI})[P \text { value }]^{\xi}\end{array}$ & & $\begin{array}{l}+2.9(+0.4,+5.4) \\
\quad[P=0.02]\end{array}$ \\
\hline \multicolumn{3}{|l|}{ Distribution of change, no. (\%) } \\
\hline 230 letter score improvement & $5(3 \%)$ & $7(5 \%)$ \\
\hline 29-25 letter score improvement & $5(3 \%)$ & $8(5 \%)$ \\
\hline 25-20 letter score improvement & $13(9 \%)$ & $15(10 \%)$ \\
\hline 19-15 letter score improvement & $16(11 \%)$ & $17(12 \%)$ \\
\hline 14-10 letter score improvement & $22(15 \%)$ & $35(24 \%)$ \\
\hline 9-5 letter score improvement & $30(21 \%)$ & $22(15 \%)$ \\
\hline Same \pm 4 letters & $31(22 \%)$ & $30(20 \%)$ \\
\hline 5-9 letters worse & $7(5 \%)$ & $5(3 \%)$ \\
\hline 10-14 letters worse & $7(5 \%)$ & $3(2 \%)$ \\
\hline$\geq 15$ letters worse & $8(6 \%)$ & $5(3 \%)$ \\
\hline $\begin{array}{l}\text { Difference in proportion with } \mathbf{1 0} \text { letter improvement from } \\
\text { ranibizumab + prompt laser treatment }(95 \% \mathrm{CI})\end{array}$ & --- & $+13 \%(+2 \%,+25 \%)$ \\
\hline Relative risk $(95 \% \mathrm{CI})$ & & $1.35(1.07,1.69)$ \\
\hline$P$ value $\mathcal{E}$ & --- & $P=0.01$ \\
\hline $\begin{array}{l}\text { Difference in proportion with } \underset{10}{ } \text { letter worsening from } \\
\text { ranibizumab + prompt laser treatment }(95 \% \mathrm{CI})\end{array}$ & --- & $-5 \%(-11 \%,+1 \%)$ \\
\hline Relative risk $(95 \% \mathrm{CI})$ & & $0.54(0.24,1.23)$ \\
\hline$P$ value $\mathcal{B}$ & --- & $P=0.14$ \\
\hline $\begin{array}{l}\text { Difference in proportion with } \ 15 \text { letter improvement from } \\
\text { ranibizumab + prompt laser treatment }(95 \% \mathrm{CI})\end{array}$ & --- & $+5 \%(-6 \%,+15 \%)$ \\
\hline Relative risk $(95 \% \mathrm{CI})$ & & $1.27(0.90,1.78)$ \\
\hline$P$ value $\mathcal{E}$ & --- & $P=0.17$ \\
\hline $\begin{array}{l}\text { Difference in proportion with } \mathbf{1} 5 \text { letter worsening from } \\
\text { ranibizumab + prompt laser treatment }(95 \% \mathrm{CI})\end{array}$ & --- & $-2 \%(-7 \%,+3 \%)$ \\
\hline Relative risk $(95 \% \mathrm{CI})$ & & $0.62(0.21,1.85)$ \\
\hline$P$ value $\mathcal{S}$ & --- & $P=0.39$ \\
\hline \multicolumn{3}{|l|}{$\mathrm{D}=$ Standard deviation; $\mathrm{CI}=$ Confidence interval. } \\
\hline \multicolumn{3}{|c|}{$\begin{array}{l}\text { * Includes only eyes with visual acuity measurements at baseline and 3-year visit. Visits occurring between } 980 \text { and } 1204 \text { days (between } 140 \text { and } \\
172 \text { weeks) from randomization were included as 3-year visits. When more than one visit occurred in this window, data from the visit closest to the } \\
\text { 3-year target date were used. }\end{array}$} \\
\hline \multicolumn{3}{|l|}{${ }^{t}$ Truncated to \pm 30 letters. } \\
\hline
\end{tabular}


